\title{
Allele-specific methylation is prevalent and is contributed by CpG-SNPs in the human genome
}

\author{
Robert Shoemaker, ${ }^{1,3}$ Jie Deng, ${ }^{2,3}$ Wei Wang, ${ }^{1,4}$ and Kun Zhang ${ }^{2,4}$ \\ ${ }^{1}$ Department of Chemistry and Biochemistry, University of California at San Diego, La Jolla, California 92093, USA; ${ }^{2}$ Department \\ of Bioengineering and Institute for Genomic Medicine, University of California at San Diego, La Jolla, California 92093, USA
}

\begin{abstract}
In diploid mammalian genomes, parental alleles can exhibit different methylation patterns (allele-specific DNA methylation, ASM), which have been documented in a small number of cases except for the imprinted regions and $\mathrm{X}$ chromosomes in females. We carried out a chromosome-wide survey of ASM across 16 human pluripotent and adult cell lines using Illumina bisulfite sequencing. We applied the principle of linkage disequilibrium (LD) analysis to characterize the correlation of methylation between adjacent $\mathrm{CpG}$ sites on single DNA molecules, and also investigated the correlation between CpG methylation and single nucleotide polymorphisms (SNPs). We observed ASM on 23\% 37\% heterozygous SNPs in any given cell line. ASM is often cell-type-specific. Furthermore, we found that a significant fraction $(38 \% \sim 88 \%)$ of ASM regions is dependent on the presence of heterozygous SNPs in CpG dinucleotides that disrupt their methylation potential. This study identified distinct types of ASM across many cell types and suggests a potential role for CpG-SNP in connecting genetic variation with the epigenome.
\end{abstract}

[Supplemental material is available online at http://www.genome.org. The sequence data from this study have been submitted to the NCBI Sequence Read Archive (http://www.ncbi.nlm.nih.gov/Traces/sra/sra.cgi) under accession no. SRA012435.]

DNA methylation is an epigenetic marker that plays a direct role in transcriptional regulation. DNA methylation patterns are tissuespecific. Embryonic stem cells undergoing differentiation show significant changes in DNA methylation patterns (Deng et al. 2009; Doi et al. 2009; Lister et al. 2009). In addition to DNA methylation pattern differences between cell lines, DNA methylation can also be allele-specific within a cell line and is thus linked to allele-specific gene expression (ASE). An example of allele-specific methylation (ASM) is genetic imprinting, which describes the parent-specific gene expression behavior of a small set of genes. The methylation pattern of imprinted genes is distinct; the inactive allele is significantly more methylated than the actively expressed allele. The number of currently known imprinting genes is suspected to be a small fraction of the total number of imprinted genes. Recent work has provided a large candidate list of imprinted genes (Luedi et al. 2007), though most of these candidates still remain to be validated. Even less is known is about the genes that fall into the broader ASM category. The biological importance of methylation is clear as disturbances of known methylation patterns are linked to disease phenotypes (Robertson 2005; Eggermann 2009).

In a recent survey of DNA methylation changes during nuclear reprogramming of human fibroblasts to induced pluripotent stem cells, $7.6 \%$ of $\mathrm{CpG}$ islands were found to be dominated by CpG sites that have intermediate levels of methylation (0.25-0.75, referred as fuzzy methylation), even though the samples used in the assay are polyclonal or monoclonal cell lines (Deng et al. 2009). A small fraction of fuzzily methylated $\mathrm{CpG}$ dinucleotides are related to $\mathrm{X}$ inactivation, and imprinting is unlikely the dominant

\footnotetext{
${ }^{3}$ These authors contributed equally to this work.

${ }^{4}$ Corresponding authors.

E-mail kzhang@bioeng.ucsd.edu.

E-mail wei-wang@ucsd.edu.

Article published online before print. Article and publication date are at http://www.genome.org/cgi/doi/10.1101/gr.104695.109.
}

effect that explains the other regions. ASM is another mechanism that could potentially explain fuzzy methylation. However, only a handful of ASM regions have been identified to date (Kerkel et al. 2008; Zhang et al. 2009b). Taking advantage of the high-resolution CpG methylation information generated from targeted bisulfite sequencing, we carried out a systematic study to characterize ASM and its role in fuzzy methylation (Supplemental Fig. 1).

\section{Results}

We reasoned that, if fuzzy methylation were due to unequal methylation levels between the two copies of the chromosomes in the same cells or the presence of heterogeneous epigenetic states among the cell population, the methylation levels on adjacent $\mathrm{CpG}$ sites of the same DNA molecules should be highly correlated. When performing Illumina sequencing on bisulfite-converted DNA, a sequencing read often contains multiple CpG sites, which can be treated as methylation haplotypes. Such methylation haplotypes are similar to SNP haplotypes, which allowed us to extend the concept of linkage disequilibrium (LD) analysis to characterizing the comethylation of CpG sites on single DNA molecules. Specifically, we used the LD measurement $r^{2}$, which indicates the fraction of variation (of methylation status) on a CpG site, A, that can be explained by the variation on another CpG site, B. Note that in this context LD is defined on a population of diploid cells.

Our analyses were based on targeted bisulfite sequencing data (41-bp reads) previously generated on 11 human pluripotent and adult cell lines (Deng et al. 2009), plus additional paired 36-bp Illumina sequencing reads on eight human cell lines (three cell lines were covered in both sets). The following regions were examined in this analysis: (1) all 2020 CpG islands on human chromosomes 12 and 20, (2) 237 promoters in eight ENCODE (the Encyclopedia of DNA Elements) regions, and (3) the 4 -kb region centered around the transcription start sites (TSS) of 26 genes related to development 
or pluripotency. In addition to the Illumina short reads, Sanger sequencing data from cloned bisulfite PCR amplicons on a selected number of regions were also generated (Supplemental Tables 1-3). Expanding on the previously published read-mapping strategy (Deng et al. 2009), we used whole-genome mapping for Illumina data and developed methylation haplotype-identifying algorithms for Illumina and Sanger sequences. Regions with at least $10 \times$ read depth were used in our LD analysis.

We first validated our LD analysis on known imprinted regions and female $\mathrm{X}$ chromosomes. In such regions we expected to observe high $r^{2}$-values that extend over a long distance. We developed an algorithm to search for such regions (see Methods). Similar to the LD blocks in the human population, we called these regions "methylation LD blocks." A block was first created between a pair of CpG sites with $r^{2}>0.3$ and extended if another CpG pair with $r^{2}>0.3$ was $<100 \mathrm{bp}$ away. Since we were most interested in examining extended organized methylatation regions, we filtered out LD blocks that spanned $<100$ bp or contained fewer than 10 CpG pairs with $r^{2}>0.3$. We observed LD blocks in all 16 cell lines for the known imprinted genes SNRPN and GNAS (Luedi et al. 2007). NNAT, another known imprinted gene, was found to contain LD blocks in 13 cell lines. There were two other imprinted genes found, but the read coverage for these genes was much lower than for SNRPN, GNAS, and NNAT. IGF2AS was covered in only three cell lines and NDN was covered in only one cell line. No LD blocks were found within these two gene regions in their respective cell lines. In female cell lines, approximately $43 \% \sim 75 \%$ of the X chromosome regions included in our analysis were covered by LD blocks, which clearly distinguished them from the majority of male lines due to $\mathrm{X}$ chromosome inactivation (Table 1). A much lower fraction $(0 \% \sim 9 \%)$ of $X$ chromosome regions was within LD blocks for the male cell lines. The only exception is Hues63, a male human embryonic stem cell line, which exhibited extended LD on the $\mathrm{X}$ chromosome. This is likely due to the presence of a subpopulation of cells that was already in the early stage of differentiation (Supplemental Fig. 2). Bisulfite Sanger sequencing of a known imprinted gene, $S N R P N$, from Hues63 revealed a methylation LD block that extended over 500 bp. In such regions, LD does not decay over the physical distance (Fig. 1A-C). However, imprinted regions are not free of noise. We also observed many pairs of CpG sites that have little or no correlation (Fig. 1B,C).

We next extended the LD analysis to the other autosomal regions. Various levels of LD were observed in all 16 cell lines included in this study (Supplemental Fig. 3). Pluripotent cell lines appear to exhibit more extended LD compared with the corresponding fibroblast lines, which can potentially be explained by the less compact chromatin structure in pluripotent cells such that cis-regulation can operate over a longer distance (Spivakov and Fisher 2007). Of the 108- to $289-\mathrm{kb}$ genomic regions with sufficient read coverage for the LD analysis, $10 \% \sim 24 \%$ were within methylation LD blocks, which vary in both the number of CpG sites per block and the block size (Table 1). The majority of the methylation LD blocks (68\% 94\%) are not known to be involved in genomic imprinting or $\mathrm{X}$ inactivation.

Many genes were found to contain LD blocks across several cell lines. Out of 309 genes that are associated with at least one LD block in at least one cell line, 100 genes (32\%) contain LD blocks in at least five cell lines. Eighteen genes (5.9\%) are found to have LD blocks in at least 12 cell lines (Supplemental Table 4). GNAS and SNRPN contain LD blocks in all 16 cell lines, and NNAT contains LD blocks in 13 cell lines. Overall, 31\% 50\% of fuzzily methylated CpGs had strong LD $\left(r^{2}>0.3\right)$ with at least one adjacent site, and $14 \% \sim 36 \%$ of fuzzily methylated regions were found within methylation LD blocks. A fraction of fuzzily methylated CpGs has neighbors with correlated methylation patterns, but many of these patterns are too localized to meet our definition for a methylation LD block. The organized CpG methylation patterns within LD blocks cannot be explained by stochastic effects. They are evidence of either epigenetic heterogeneity across cells or preferential methylation of one particular parental chromosome copy.

To explore whether the methylation LD blocks are due to heterogeneity of cell populations or unequal methylation of chromosome copies, we developed an algorithm for SNP calling from bisulfite sequencing reads (see Methods). Heterozygous SNPs allowed us to distinguish methylation haplotypes from the two parents, and to detect allele-specific methylation. If fuzzy methylation was simply due to the presence of heterogeneity in cell populations, we would not expect to observe allelic preference within a cell. Due

Table 1. LD $r^{2}$ statistics

\begin{tabular}{|c|c|c|c|c|c|}
\hline Cell line (gender) & $\begin{array}{l}\text { Total regions } \\
\text { analyzed (bp) }\end{array}$ & $\begin{array}{l}\text { Percent of LD } \\
\text { block covered }^{b}\end{array}$ & $\begin{array}{l}\text { Chr X regions } \\
\text { analyzed (bp) }\end{array}$ & $\begin{array}{l}\text { Chr X percent of } \\
\text { LD block covered }\end{array}$ & $\begin{array}{l}\text { Percent of LD blocks not in } \\
\text { chr } \mathrm{X} \text { or known imprinted genes }\end{array}$ \\
\hline $\mathrm{BJ}(\mathrm{M})$ & 288,574 & $11.99 \%$ & 4560 & $0 \%$ & $84.03 \%$ \\
\hline BJ-iPS11 (M) & 117,147 & $21.73 \%$ & 747 & $0 \%$ & $87.46 \%$ \\
\hline BJ-iPS12 (M) & 118,334 & $22.36 \%$ & 986 & $0 \%$ & $85.76 \%$ \\
\hline hFib2 (M) & 119,085 & $16.84 \%$ & 1411 & $0 \%$ & $85.96 \%$ \\
\hline hFib2-iPS (M) & 129,883 & $13.52 \%$ & 1172 & $0 \%$ & $86.32 \%$ \\
\hline Hues12 (F) & 246,680 & $17.17 \%$ & 9006 & $57 \%$ & $79.20 \%$ \\
\hline Hues42 (M) & 126,481 & $15.92 \%$ & 929 & $0 \%$ & $85.38 \%$ \\
\hline Hues63 (M) & 152,400 & $19.47 \%$ & 5616 & $71.08 \%$ & $71.09 \%$ \\
\hline Hybrid_1 (M/F) & 246,159 & $15.75 \%$ & 6406 & $47.35 \%$ & $81.94 \%$ \\
\hline IMR90 (F) & 200,953 & $10.35 \%$ & 7815 & $42.47 \%$ & $68.02 \%$ \\
\hline IMR90-iPS (F) & 107,727 & $21.75 \%$ & 4428 & $74.82 \%$ & $73.71 \%$ \\
\hline PGP1F (M) & 229,597 & $15.63 \%$ & 4394 & $2.62 \%$ & $87.18 \%$ \\
\hline PGP1-iPS1 (M) & 171,338 & $24.21 \%$ & 2128 & $8.74 \%$ & $93.47 \%$ \\
\hline PGP1L (M) & 219,643 & $16.09 \%$ & 2386 & $8.17 \%$ & $91.11 \%$ \\
\hline PGP3L (F) & 245,052 & $21.22 \%$ & 10,634 & $38.83 \%$ & $85.17 \%$ \\
\hline PGP9L (F) & 247,465 & $21.24 \%$ & 10,924 & $47.11 \%$ & $81.56 \%$ \\
\hline
\end{tabular}

ancludes the base pairs between CpGs that had sufficient read coverage for the LD analysis.

${ }^{b}$ LD blocks are defined as regions that span $>100 \mathrm{bp}$ and contain $>10 \mathrm{CpG}$ dinucleotide pairs whose $r^{2}>0.3$. Female cell lines, containing two $X$ chromosomes, are expected to show LD blocks in regions of $X$ chromosome inactivation.

CPercentage of LD blocks not located in chromosome $X$ or known imprinting genes.

\section{Genome Research}


A

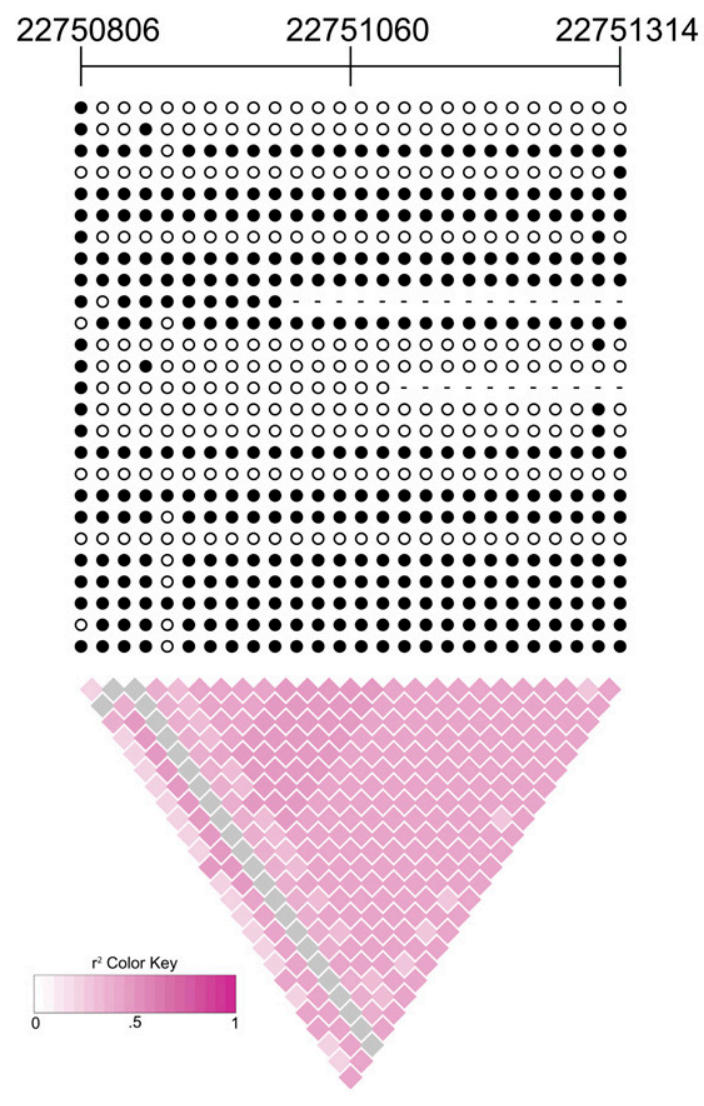

B

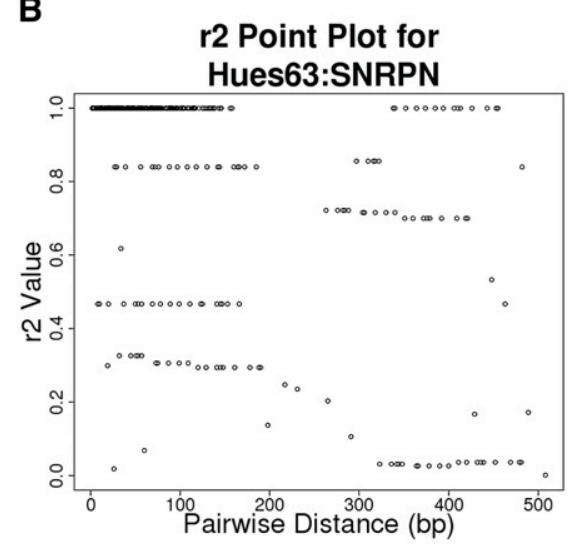

C

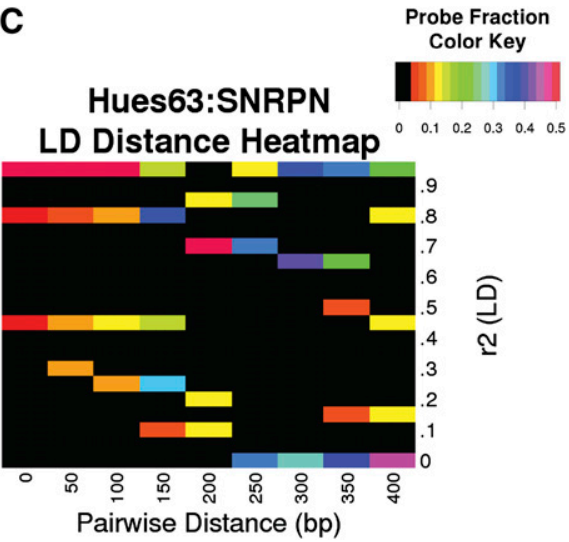

Figure 1. Linkage disequilibrium (LD) analysis of $\mathrm{CpG}$ methylation haplotypes. (A) LD diagram of $5^{\prime}$ region of the imprinted gene SNRPN (chr15:22750806-22751314) from Hues63. Each row represents a Sanger sequence and each column represents a CpG dinucleotide. (Filled circles) Methylated CpGs; (open circles) unmethylated CpGs, (dashed lines) methylation state of the CpG could not be determined. Chromosomal coordinates are listed above. This region shows a methylation LD block spanned over $500 \mathrm{bp}$ in Sanger reads. $(B, C)$ CpG pairwise $r^{2}$-value plots and heatmap for $5^{\prime}$ region of the SNRPN gene. While the majority of CpG pairs have high methylation correlation values $\left(r^{2}>0.3\right)$, some pairs of CpG sites have little or no correlation $\left(r^{2}<0.3\right)$. The pairwise distance represents the separation of the CpG dinucleotides used in the $r^{2}$ calculation. The heatmap colors represent the probe fraction at a given pairwise distance (rounded down to the nearest $50 \mathrm{bp}$ ) that has the indicated $r^{2}$-value. The probe fractions for each pairwise distance sum to one. The color scale saturates at 0.5 , so that small probe fraction differences can be distinguished.

to the reduced sequence complexity of the bisulfite-converted genome, we also adapted SAMtools to make SNP calls (Li et al. 2009a). We compiled a list of confident SNP calls based on the intersection between our own algorithm and SAMtools. In total, we identified 240-457 heterozygous SNPs and 197-391 homozygous SNPs in each cell line (Supplemental Table 5). The three cell lines PGP1L, PGPF, and PGP1-iPS were derived from the same individual (PGP1) in the Personal Genome Project, whose full genome was recently sequenced (Drmanac et al. 2010). We observed a high concordance between the SNPs called from our bisulfite sequencing data and those generated by whole-genome sequencing. For the PGP1 lines, 96\%-97\% of SNP calls made by Complete Genomics matched our SNP calls. As expected, cell lines of identical genetic background showed an expected higher number of pairwise overlapped SNP calls relative to the other cell lines (Supplemental Tables 6,7). From the bisulfite sequencing reads that contain both heterozygous SNPs and at least one CpG site, we identified methylation that significantly associates with one allele of a SNP using the Fisher's exact test (see Methods). We also required a minimum methylation frequency difference of 0.1 between the alleles for ASM categorization. In each cell line, $23 \% \sim 37 \%$ of heterozygous SNPs were found to associate with ASM (Table 2). Only a small fraction of ASM (6\%) was consistent across all cell lines in which the heterozygous SNPs are present. The remaining cases are either cell-type-specific or individualspecific (Supplemental Fig. 4). We also compared the allelic methylation frequencies of 980 individual CpG sites that are linked to SNPs from two batches of IMR90 fibroblast cultures. Excellent correlation was observed between the biological replicates (Pearson correlation coefficient $r^{2}=0.90$ ), which indicated that our observations were not due to technical artifacts or biological fluctuations.

We validated 12 SNP sites by performing bisulfite PCR, cloning, and Sanger sequencing on one or more cell lines in which SNPs were called. A total of 21 SNP regions were amplified and Sanger sequenced. The Sanger regions that show ASM fall into two categories. In category I, more than one adjacent $\mathrm{CpG}$ site exhibit consistent bias in methylation. The known imprinted regions fall into this category. However, even in autosomal regions not known to be related to genetic imprinting, similar allelic preference can extend over 900 bp (Fig. 2A,B; Supplemental Fig. 1A,B). In category II, ASM is highly localized and restricted to only a very small number of CpG sites in a region (Fig. 2C,D; Supplemental Fig. 5). Seven sequences had inconsistent ASM classification between the 
Table 2. SNP calling and ASM categorization statistics

\begin{tabular}{|c|c|c|c|c|c|c|}
\hline Cell line & $\begin{array}{l}\text { Heterozygous } \\
\text { SNPs called }\end{array}$ & $\begin{array}{l}\text { No. of ASM } \\
\text { regions }\end{array}$ & $\begin{array}{l}\text { No. of ASM regions } \\
\text { with CpG-SNPs }\end{array}$ & $\begin{array}{c}\text { Percent of } \\
\text { category I ASM }\end{array}$ & $\begin{array}{c}\text { Percent of category I } \\
\text { ASM containing CpG-SNPs }\end{array}$ & $\begin{array}{c}\text { Percent of } \\
\text { category II ASM }\end{array}$ \\
\hline BJ & 457 & 123 & 102 & $11 \%$ & $59 \%$ & $16 \%$ \\
\hline B]-iPS11 & 381 & 102 & 92 & $5 \%$ & $70 \%$ & $22 \%$ \\
\hline B]-iPS12 & 402 & 108 & 96 & $7 \%$ & $61 \%$ & $20 \%$ \\
\hline hFib2 & 279 & 87 & 58 & $19 \%$ & $46 \%$ & $12 \%$ \\
\hline hFib2-iPS & 283 & 67 & 58 & $7 \%$ & $53 \%$ & $17 \%$ \\
\hline Hues 12 & 391 & 114 & 97 & $7 \%$ & $45 \%$ & $22 \%$ \\
\hline Hues42 & 308 & 76 & 63 & $7 \%$ & $52 \%$ & $17 \%$ \\
\hline Hues63 & 382 & 89 & 76 & $5 \%$ & $55 \%$ & $18 \%$ \\
\hline Hybrid_1 & 395 & 120 & 107 & $8 \%$ & $67 \%$ & $22 \%$ \\
\hline IMR90 & 436 & 162 & 128 & $22 \%$ & $67 \%$ & $15 \%$ \\
\hline IMR90-iPS & 430 & 129 & 120 & $3 \%$ & $60 \%$ & $27 \%$ \\
\hline PGP1_F & 292 & 83 & 66 & $12 \%$ & $59 \%$ & $17 \%$ \\
\hline PGP1-iPS1 & 240 & 62 & 56 & $9 \%$ & $77 \%$ & $17 \%$ \\
\hline PGP1L & 257 & 88 & 61 & $20 \%$ & $59 \%$ & $14 \%$ \\
\hline PGP3L & 254 & 94 & 62 & $20 \%$ & $51 \%$ & $17 \%$ \\
\hline PGP9L & 272 & 74 & 61 & $8 \%$ & $57 \%$ & $19 \%$ \\
\hline
\end{tabular}

Category I SNPs include SNPs whose ASM is not based solely on SNP-containing CpG sites. However, many category I SNP regions still have SNPcontaining CpG nucleotides. Category II SNPs include SNPs whose ASM is completely dependent on the presence of a SNP-containing CpG dinucleotide. Category III SNPs (data not shown) do not show ASM. The category I and category II percentages represent the number of SNPs in the respective category divided by the total number of heterozygous SNP calls in each cell line.

Illumina and Sanger sequencing. Four of these inconsistencies were due to the inability to establish statistical ASM significance due to the low Sanger sequencing read depth. Two SNP sites predicted by the Illumina data were not found in the Sanger data (Supplemental Table 1), which are likely due to incorrect mapping of short bisulfite sequencing reads. One SNP site had inconsistent ASM behavior between Sanger and Illumina data. Overall, we found a Pearson correlation coefficient of $r^{2}=0.78$ for the allelic methylation frequencies of CpGs shared between the Sanger sequencing and Illumina data sets.

Next, we explored potential cis-regulatory mechanisms that account for ASM. We identified many ASM regions that contained at least one heterozygous SNP that overlapped with a CpG dinucleotide. As an example, the region containing the T/C SNP site rs10846023 in the BJ-iPS12 cell line shows ASM that spans over $500 \mathrm{bp}$. This SNP is present at a CG dinucleotide, and the T allele disrupts the CG site. However, the ASM behavior at this site is also exhibited in nearby sites (Fig. 2A,B), which suggests that some uncharacterized regulatory mechanisms determine the ASM and such regulation may be dependent on the local sequence context in cis. We also found examples of specific methylation behavior that did not correlate with the base identity of a nearby SNP (Supplemental Fig. 6). Given the presence of SNP-containing CpGs, we refined our ASM categories so that category I represented ASM that was not solely dependent on SNP-containing CpGs. Category II represented ASM that solely depended on SNP-containing CpGs. We found $45 \%-77 \%$ of category I SNP regions have SNPs at CpG dinucleotides, which suggests that SNPs on CpG sites may have an impact on differential methylation establishment if the SNPs are located in regions where methylation regulators are involved. In contrast are the regions where the ASM affects only CpG locations overlapping with SNPs. One allele of such SNPs eliminates the CpG sites, thus preventing them from being methylated. One example is the region around SNP site rs6061990 in PGP3L (Supplemental Fig. 5) and PGP9L (Fig. 2C,D). The PGP9L cell line shows ASM at two CpG dinucleotides while the region in PGP3L shows ASM at one CpG site. This example demonstrates that individual ASM sites can be dependent on sequence difference alone. A complete list of ASM categorizations of SNP regions is available on our supplemental web- site (http://genome-tech.ucsd.edu/public/ASM/) and in the Supplemental materials. Overall, $38 \% \sim 88 \%$ of ASM regions are solely due to the presence of SNPs at CpG dinucleotides, revealing that genetic variation at $\mathrm{CpG}$ sites is a dominating factor for ASM.

Finally, ASM regions that span across multiple CpG sites are likely regulated by other cis-regulatory mechanisms. We found many cases where the methylation state closely correlated with the alleles identified by a SNP. Such behavior is more likely explained by an allele-specific regulatory mechanism rather than cell subpopulations undergoing different epigenetic processes. For example, regions that exhibit reverse allelic preference in different cell lines of the same genetic background are observed (Supplemental Fig. 7), which can only be explained by a regulatory mechanism that involves more than one cis-regulator with opposite effects. Out of the category I ASM regions that are covered by our LD analysis, $8 \%-35 \%$ are within methylation LD blocks, demonstrating that ASM analysis uniquely identified new regions where methylation was due to allele specific cis-regulation. Finally, the two IMR90 biological replicates showed that $83 \%$ of ASM calls were consistent in both experiments (201 matched ASM SNP calls out of 242 total ASM SNP calls). Therefore, ASM is due to biological regulation instead of biological noise or technical artifacts.

\section{Discussion}

We set out in this analysis to investigate the basis of fuzzy methylation with two novel approaches: adapting linkage disequilibrium analysis to methylation data, and performing SNP calling on bisulfite sequencing reads. This study represents the largest survey of ASM in the human genome to date. Hundreds of methylation LD blocks were identified from over $2000 \mathrm{CpG}$ islands in two human chromosomes and dozens of other regions. Roughly 30\% 48\% of fuzzily methylated CpGs were found to have $r^{2}>0.3$ and 14\% 36\% of fuzzily methylated CpGs were found within LD blocks. This shows stochastic effects do not explain a significant amount of observed fuzzy methylation. Our SNP ASM analysis found that 23\%-37\% of heterozygous SNPs are associated with ASM. The frequency of ASM is similar to the frequency of allele-specific gene expression (ASE) observed in the human genome (Yan et al. 2002; Ge et al. 2009; Lee 
A

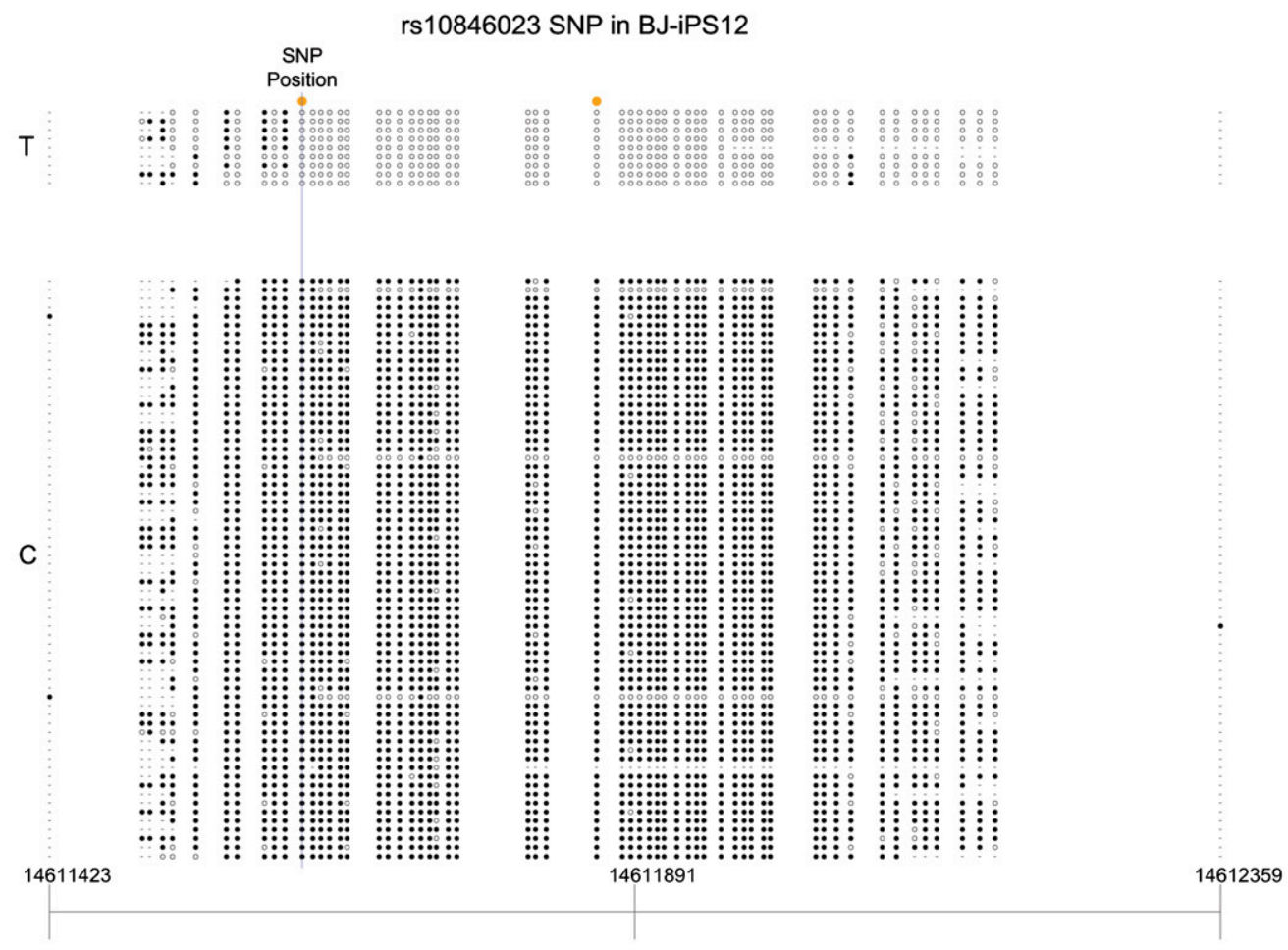

B

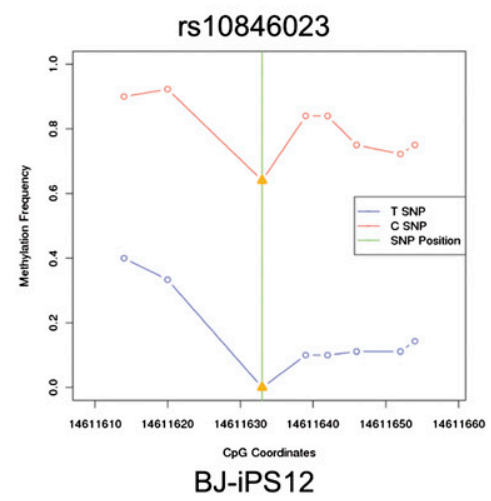

C
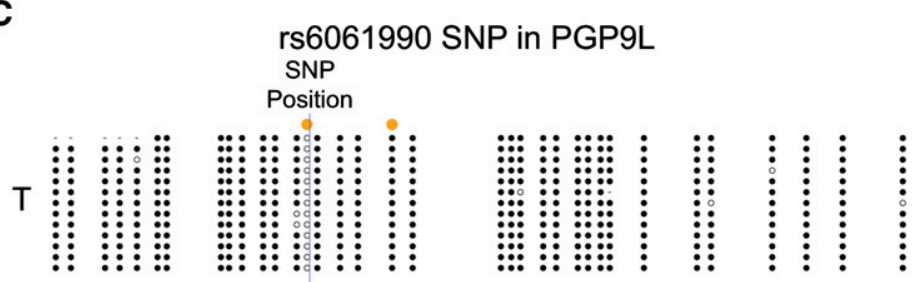

D
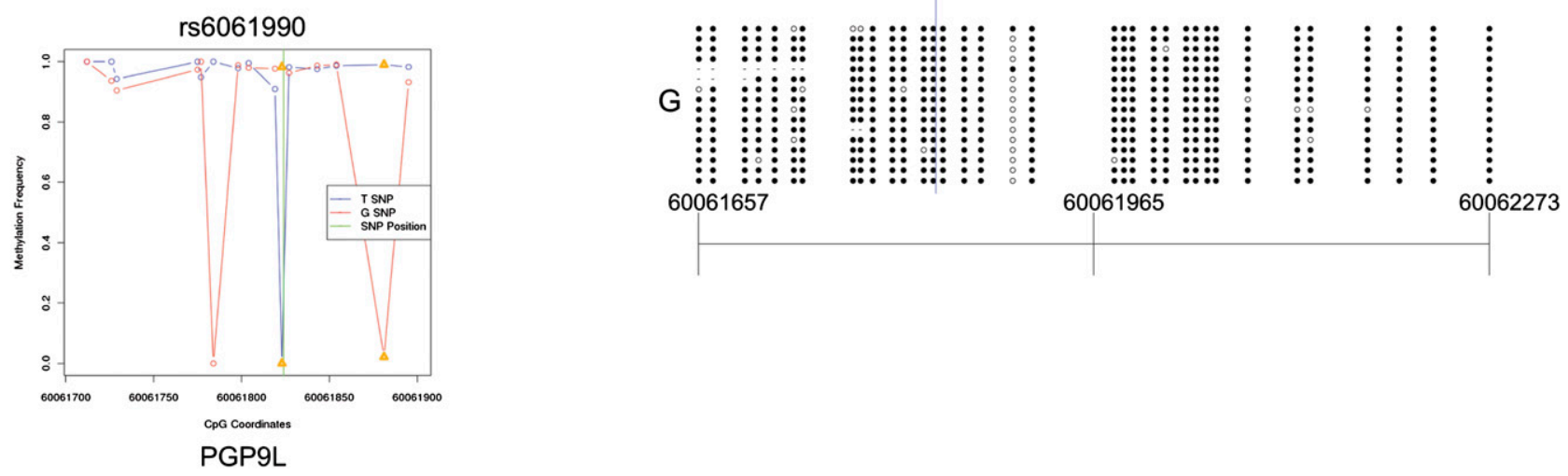

Figure 2. (Legend on next page) 
et al. 2009; Zhang et al. 2009a; Heap et al. 2010). ASE has been considered as an important indicator for the presence of functional cisregulatory variants (Pastinen and Hudson 2004). ASE and ASM could be tightly coupled by the same cis-regulatory variants (Ghotbi et al. 2009; Milani et al. 2009). Similar to gene expression, methylation or ASM can be considered as quantitative traits for population genomic analysis. One important finding of this work is that a SNP could be a functional cis-regulatory variant by disrupting a $\mathrm{CpG}$ methylation site. According to the snp129 database, there are 225,659 known SNPs that locate on CpG sites. In addition, because CpG dinucleotides are highly mutable, there are many CpG rare variants in individual genomes (Li et al. 2009b). CpG SNPs are likely an important class of cis-regulatory polymorphisms that connects genetic variation to the individual variability of the epigenome.

\section{Methods}

\section{Bisulfite targeted reseqeuncing with padlock probes}

Bisulfite padlock capture and targeted resequencing were performed as described (Deng et al. 2009). Briefly, genomic DNA was extracted from frozen pellets of lymphocyte, fibroblast, iPS, or hES cells using Qiagen DNeasy columns, and bisulfite converted with Zymo DNA methylation Gold kit (Zymo research). The Cpg30k padlock probe library was annealed to the bisulfite-converted sample DNAs, circularized, and amplified by PCR. Random shotgun sequencing library was generated by USER/MmeI enzymatic fragmentation from the amplicons of captured targets, which then were subsequently sequenced by Illumina Genome Analyzer.

\section{Bisulfite PCR and cloning for Sanger sequencing}

Bisulfite PCR reactions were performed in $100-\mu \mathrm{L}$ reactions including $50 \mathrm{ng}$ of bisulfite-converted genomic DNA, $200 \mu \mathrm{M}$ dNTP, $0.4 \mu \mathrm{M}$ forward and reverse PCR primers, and $1 \times$ IQ PCR Supermix (Bio-Rad) for $2 \mathrm{~min}$ at $94^{\circ} \mathrm{C} ; 45$ cycles of $30 \mathrm{sec}$ at $94^{\circ} \mathrm{C}, 1 \mathrm{~min}$ at $62^{\circ} \mathrm{C}, 1 \mathrm{~min}$ at $72^{\circ} \mathrm{C}$; and finally $5 \mathrm{~min}$ at $72^{\circ} \mathrm{C}$. Bisulfite PCR products were cloned in the pCR 2.1-TOPO vector (Invitrogen), and multiple clones were picked and sequenced at Agencourt. The primer sequences are in Supplemental Table 2.

\section{Statistical analysis}

Raw Illumina sequencing reads from the previously published data set and the new paired-end data set were combined to produce a data set that consisted of 16 cell lines (Deng et al. 2009). These combined reads were mapped to the in silico bisulfite-converted human genome sequence (hg18) via SOAP2 (Li et al. 2009c). Mates from paired-end reads were mapped independently. Reads were allowed to have up to two mismatches, and reads that mapped to multiple locations were excluded. Sanger reads were mapped analogously except that UCSC BLAT (Kent 2002) was used instead of SOAP to map these sequences to a reference template. Due to the longer length of Sanger sequences and their known position in the genome, Sanger sequence alignments were allowed to have multiple mismatches and gaps.

SNPs were called with an algorithm that assigned probabilities to each genotype. Bisulfite-converted strands were analyzed independently, and the probability of each genotype was assessed via the Fisher's exact test. SNP calls were limited to annotated dbSNP 129 sites, and quality score filtering was applied to filter out low-quality base calls. There was a $10 \times$ minimum read depth per strand requirement for SNP calling. SNP calls were also made with SAMtools, and the intersection of SNP calls between our algorithm and SAMtools was used in further analyses. SNP sites were grouped into three categories: (1) SNPs showing either CpG-specific or averaged ASM that is independent of SNPs at CG sites, (2) SNPs showing ASM that is dependent on the presence of a SNP CpG overlap, and (3) SNPs that do not show ASM. In order for a CpG site or SNP region to be classified as ASM in the Illumina data, it needed to have an allelic methylation frequency difference of at least 0.1. Sanger sequence ASM labeling used the same statistical test as described above without the allelic methylation frequency difference requirement due to the lower read coverage.

ASM was determined by creating a $2 \times 2$ contingency table where the two columns represented the two alleles identified by a SNP. The two rows represented the counts of methylated and unmethylated cytosines at CpG site(s) located on a SNP-containing read. Each $\mathrm{CpG}$ site was treated independently for $\mathrm{CpG}$-specific ASM, and for average ASM the methylated and unmethylated cytosine counts were summed across CpG sites allele, specifically. If a SNP region contained SNPs at CpG sites, the average ASM calculation was repeated while excluding SNP-containing CpG sites.

Reads containing two or more CpG sites were used for the linkage disequilibrium $r^{2}$ analysis. A CpG pair needed to have a minimum $10 \times$ read depth. LD $r^{2}$-values were considered high if they were greater than 0.3. For the Illumina data, an LD block was created if a CpG pair had a high $r^{2}$-value. This region was expanded if there was an overlapping CpG pair with a high $r^{2}$-value or a CpG pair within 100 bp with a high $r^{2}$-value. LD blocks span at least $100 \mathrm{bp}$ and contain at least $10 \mathrm{CpG}$ pairs with $r^{2}>0.3$.

All raw data, SNP calls with ASM categorizations, LD analyses, Sanger diagrams, and methylation frequency data are available at http://genome-tech.ucsd.edu/public/ASM/ and in the Supplemental materials. The raw Illumina sequences are available at the NCBI Sequence Read Archive (accession no. SRA012435).

\section{Acknowledgments}

We thank the laboratories of George Church, George Daley, Kevin Eggan, Konrad Hochedlinger, and James Thomson for providing DNA samples, and the UCSD BioGem Core facility for assistance with Illumina sequencing. This study is supported by the UCSD new faculty startup fund, NIH/NIDA R01-DA025779 (K.Z.), and NIH R01GM072856 (W.W.). J.D. was sponsored by a CIRM postdoctoral fellowship.

Author contributions: K.Z. and W.W. oversaw the project. J.D. performed padlock bisulfite sequencing and various validation

Figure 2. Allele-specific methylation. $(A)$ Sanger reads from BJ-iPS12 show an extended ASM region in dbSNP 129 rs10846023 indexed region (chr12:14611423-14612359) in the intron of the RAB1 1FIP1 (also known as FL)22622) gene. This ASM region includes two CpG dinucleotides that overlap with SNPs, but the ASM is not limited to these sites. (Orange circles) SNPs that overlap with CpG dinucleotides. (B) An allele-specific methylation frequency graph based on aligned Illumina data showing ASM at rs10846023 in BJ-iPS12 (chr12:14611616-14611654). ( $Y$-axis) Methylation frequency, where a value of 1 indicates complete methylation at a CpG dinucleotide; ( $x$-axis) chromosomal coordinates. (C) Sanger sequence data around SNP site rs6061990 (TAF4 intronic region, chr20:60061657-60062273) in PGP9L illustrates an example where ASM is solely dependent on SNPs at CpG dinucleotides. (D) An allele-specific methylation frequency graph based on Illumina data showing ASM at rs6061990 in PGP9L (chr20:6006171260061895). (Green line) SNP position; (orange triangles) SNPs that overlap with CpG dinucleotides. Note that the Illumina data show ASM at a CpG (chr20:60061784) that is not supported by the Sanger data.

\section{Genome Research}


assays. R.S. performed algorithm development and bioinformatics analysis. R.S., J.D., W.W., and K.Z wrote the manuscript.

\section{References}

Deng J, Shoemaker R, Xie B, Gore A, LeProust EM, Antosiewicz-Bourget J, Egli D, Maherali N, Park IH, Yu J, et al. 2009. Targeted bisulfite sequencing reveals changes in DNA methylation associated with nuclear reprogramming. Nat Biotechnol 27: 353-360.

Doi A, Park IH, Wen B, Murakami P, Aryee MJ, Irizarry R, Herb B, Ladd-Acosta C, Rho J, Loewer S, et al. 2009. Differential methylation of tissue- and cancer-specific CpG island shores distinguishes human induced pluripotent stem cells, embryonic stem cells and fibroblasts. Nat Genet 41: 1350-1353.

Drmanac R, Sparks AB, Callow MJ, Halpern AL, Burns NL, Kermani BG, Carnevali P, Nazarenko I, Nilsen GB, Yeung G, et al. 2010. Human genome sequencing using unchained base reads on self-assembling DNA nanoarrays. Science 327: 78-81.

Eggermann T. 2009. Silver-Russell and Beckwith-Wiedemann syndromes: Opposite (epi)mutations in 11 p15 result in opposite clinical pictures. Horm Res 71 (Suppl 2): 30-35.

Ge B, Pokholok DK, Kwan T, Grundberg E, Morcos L, Verlaan DJ, Le J, Koka V, Lam KC, Gagne V, et al. 2009. Global patterns of cis variation in human cells revealed by high-density allelic expression analysis. Nat Genet 41: 1216-1222.

Ghotbi R, Gomez A, Milani L, Tybring G, Syvanen AC, Bertilsson L, Ingelman-Sundberg M, Aklillu E. 2009. Allele-specific expression and gene methylation in the control of CYP1A2 mRNA level in human livers. Pharmacogenomics J 9: 208-217.

Heap GA, Yang JH, Downes K, Healy BC, Hunt KA, Bockett N, Franke L, Dubois PC, Mein CA, Dobson RJ, et al. 2010. Genome-wide analysis of allelic expression imbalance in human primary cells by high throughput transcriptome resequencing. Hum Mol Genet 19: 122-134.

Kent WJ. 2002. BLAT-the BLAST-like alignment tool. Genome Res 12: 656664.

Kerkel K, Spadola A, Yuan E, Kosek J, Jiang L, Hod E, Li K, Murty VV, Schupf $\mathrm{N}$, Vilain E, et al. 2008. Genomic surveys by methylation-sensitive SNP analysis identify sequence-dependent allele-specific DNA methylation. Nat Genet 40: 904-908.

Lee JH, Park IH, Gao Y, Li JB, Li Z, Daley GQ, Zhang K, Church GM. 2009. A robust approach to identifying tissue-specific gene expression regulatory variants using personalized human induced pluripotent stem cells. PLoS Genet 5: e1000718. doi: 10.1371/journal.pgen.1000718.

Li H, Handsaker B, Wysoker A, Fennell T, Ruan J, Homer N, Marth G, Abecasis G, Durbin R. 2009a. The Sequence Alignment/Map (SAM) format and SAMtools. Bioinformatics 25: 2078-2079.

Li JB, Gao Y, Aach J, Zhang K, Kryukov GV, Xie B, Ahlford A, Yoon JK, Rosenbaum AM, Zaranek AW, et al. 2009b. Multiplex padlock targeted sequencing reveals human hypermutable CpG variations. Genome Res 19: 1606-1615.

Li R, Yu C, Li Y, Lam TW, Yiu SM, Kristiansen K, Wang J. 2009c. SOAP2: An improved ultrafast tool for short read alignment. Bioinformatics 25: 1966-1967.

Lister R, Pelizzola M, Dowen RH, Hawkins RD, Hon G, Tonti-Filippini J, Nery JR, Lee L, Ye Z, Ngo QM, et al. 2009. Human DNA methylomes at base resolution show widespread epigenomic differences. Nature 462: 315322 .

Luedi PP, Dietrich FS, Weidman JR, Bosko JM, Jirtle RL, Hartemink AJ. 2007. Computational and experimental identification of novel human imprinted genes. Genome Res 17: 1723-1730.

Milani L, Lundmark A, Nordlund J, Kiialainen A, Flaegstad T, Jonmundsson G, Kanerva J, Schmiegelow K, Gunderson KL, Lonnerholm G, et al. 2009. Allele-specific gene expression patterns in primary leukemic cells reveal regulation of gene expression by $\mathrm{CpG}$ site methylation. Genome Res 19: 1-11.

Pastinen T, Hudson TJ. 2004. Cis-acting regulatory variation in the human genome. Science 306: 647-650.

Robertson KD. 2005. DNA methylation and human disease. Nat Rev Genet 6: $597-610$.

Spivakov M, Fisher AG. 2007. Epigenetic signatures of stem-cell identity. Nat Rev Genet 8: 263-271.

Yan H, Yuan W, Velculescu VE, Vogelstein B, Kinzler KW. 2002. Allelic variation in human gene expression. Science 297: 1143.

Zhang K, Li JB, Gao Y, Egli D, Xie B, Deng J, Li Z, Lee JH, Aach J, Leproust EM, et al. 2009a. Digital RNA allelotyping reveals tissue-specific and allele-specific gene expression in human. Nat Methods 6: 613-618.

Zhang Y, Rohde C, Reinhardt R, Voelcker-Rehage C, Jeltsch A. 2009b. Non-imprinted allele-specific DNA methylation on human autosomes. Genome Biol 10: R138. doi: 10.1186/gb-2009-10-12-r138.

Received December 29, 2009; accepted in revised form April 15, 2010. 


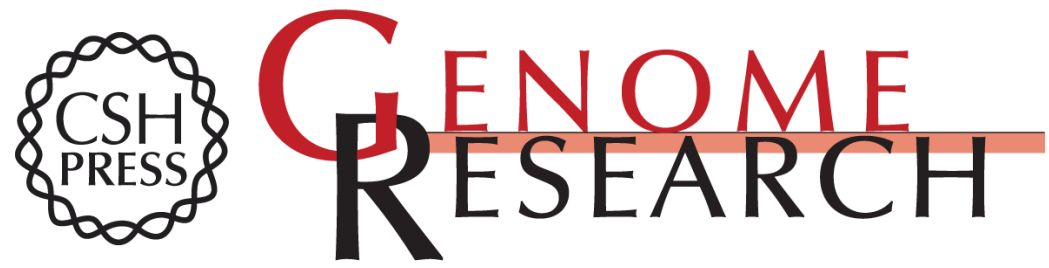

\section{Allele-specific methylation is prevalent and is contributed by CpG-SNPs in the human genome}

Robert Shoemaker, Jie Deng, Wei Wang, et al.

Genome Res. 2010 20: 883-889 originally published online April 23, 2010

Access the most recent version at doi:10.1101/gr.104695.109

Supplemental Material

References

License

Email Alerting Service
http://genome.cshlp.org/content/suppl/2010/04/22/gr.104695.109.DC1

This article cites 22 articles, 7 of which can be accessed free at: http://genome.cshlp.org/content/20/7/883.full.html\#ref-list-1

Receive free email alerts when new articles cite this article - sign up in the box at the top right corner of the article or click here.

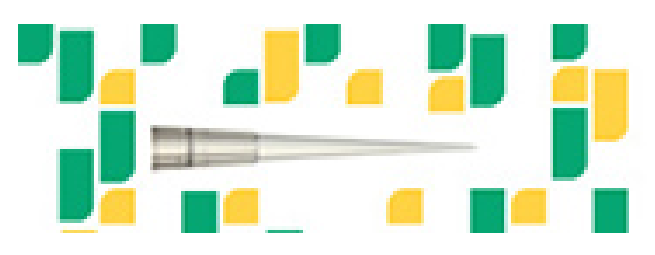

Focused on your science.

\section{Jכん,}

SCIENTIFIC

suos or seisnes

To subscribe to Genome Research go to: https://genome.cshlp.org/subscriptions 